\title{
Bilateral intracranial subdural osteomas of varying sizes imaged 7 years apart: illustrative case
}

\author{
Fahad J. Laghari, MD, ${ }^{1}$ Sarah Eakin, MD, ${ }^{2}$ and Salem El-Zuway, MD, FRCSC ${ }^{3}$ \\ Divisions of ${ }^{1}$ Neurology, ${ }^{2}$ Pathology, and ${ }^{3}$ Neurosurgery, University of Pittsburgh Medical Center Hamot, Erie, Pennsylvania
}

\begin{abstract}
BACKGROUND Previously, solitary and unilateral aggregates of intracranial subdural osteomas have been described. These tumors are thought to be slow growing and at times inconsequential on the basis of characteristics of subjacent brain. Unilateral location and history of traumas have led to the thought that the head trauma may play a role in pathogenesis.

OBSERVATIONS The authors describe a unique case of a patient who was found to have bilateral intracranial subdural osteomas of unequal size on the basis of computed tomography and magnetic resonance imaging. The presenting symptom was headache. Initially small and thought to be irrelevant, these tumors grew over the course of 7 years to cause mass effect and effacement of the sulci and gyri. The larger 15-cm-long tumor was excised and was sent for pathology, which showed classic histology for subdural osteoma.

LESSONS The described case uniquely demonstrates evidence of the slow growth of intracranial subdural osteomas over the course of years. It is crucial not to disregard the tumor because it can grow over time to cause mass effect. Patient follow-up is strongly recommended. Bilateral tumor occurrence at a similar location in this case supports an etiology other than trauma. Further research is necessary.
\end{abstract}

https://thejns.org/doi/abs/10.3171/CASE20141

KEYWORDS intracranial; subdural osteoma; intracranial bony tumor

Intracranial subdural osteomas are rare bony tumors that have no attachment to the overlying skull. Headache is the most frequent presentation. Previous case reports describe tumors that are mostly solitary or unilateral aggregates at one location. ${ }^{1}$ We present a unique case with slow-growing bilateral lesions neuroimaged 7 years apart. The larger 15-cm-long tumor on the left was excised, and the biopsy showed intracranial subdural osteoma.

\section{Illustrative Case}

\section{Presentation}

A 35-year-old woman with a past medical history of congenital hearing loss had been followed up in the neurology clinic for a prolonged history of headaches. She had first presented 7 years earlier with headaches localized to the left frontotemporal region. At her initial presentation, magnetic resonance imaging (MRI) of her brain depicted a mass lesion measuring $1.3 \mathrm{~cm}$ in thickness and $5 \mathrm{~cm}$ in length that was initially interpreted as a subdural hematoma in the left frontoparietal region. There was no associated vasogenic edema, mass effect, or effacement of adjacent sulci or gyri, pointing toward chronicity of the mass (Fig. 1A). Head computed tomography (CT) was performed to rule out blood, and the result was more consistent with a bony lesion (Fig. 1B); it also showed a similar, smaller bony lesion in the right frontal region (Fig. 1C).

The patient had been managed conservatively with watchful observation with repeat serial neuroimaging. Subsequently, her headache symptoms were occasional and infrequent. Seven years after her initial presentation, the patient had a fall that led to a flare-up of her preexisting headache, lasting months, which led her treating physicians to repeat neuroimaging. CT of the head and MRI of the brain both showed further significant interval growth of the left mass lesion, with significant mass effect to the subjacent brain parenchyma with effacement of the sulci and gyri. The imaging also showed some inconsequential growth of the mass on the right (Fig. 2).

\section{Operative Intervention}

Because of the tumor's growth and its effects on the brain parenchyma on the left side, operative tumor resection was suggested. 

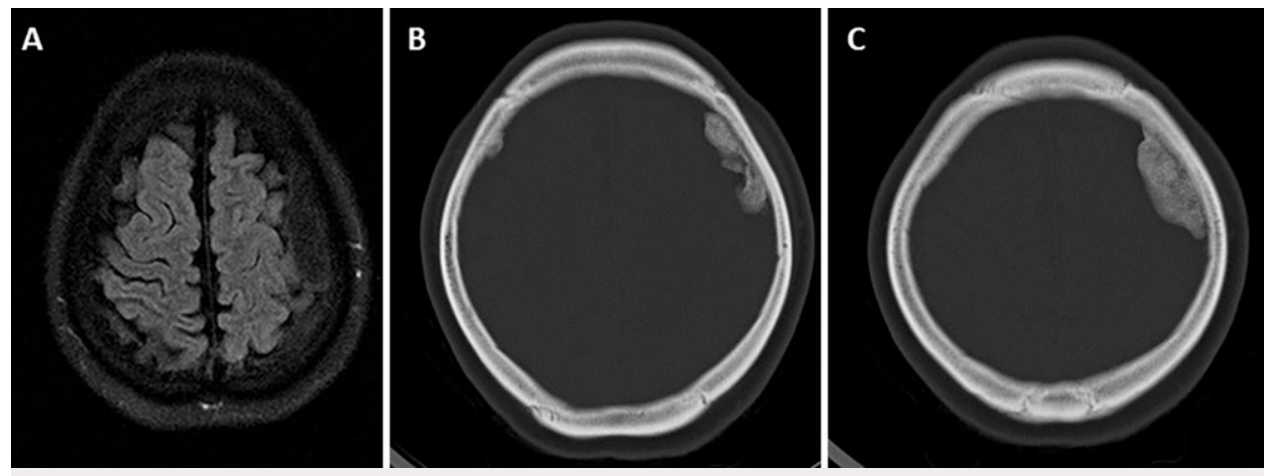

FIG. 1. Initial imaging revealed different-sized bony lesions on the left and right. A: Fluid-attenuated inversion recovery MRI of the brain shows a longitudinal mass on the left. B: Head CT shows a protuberant mass on the left with a density similar to that of the overlying bone. $\mathrm{C}$ : A small bony lesion is shown on the right.

The patient underwent a left frontotemporoparietal craniotomy with resection of the tumor. During the operation, it was found that the overlying skull flap was unaffected by the neoplastic process, and there was no tumor invasion to the overlying skull and meninges. Underneath the dura, a 15-cm, large, bony mass lesion with imprints of gyri and sulci was removed en bloc. It was hard in consistency and adherent to the overlying dura. Grossly, the tumor was not invading the underlying brain parenchyma, and there was a clear plane in between (Fig. 3).

\section{Pathology}

The histopathology of the tumor showed viable, lamellated, mature trabecular bone. The underlying normocellular marrow showed trilineage hematopoiesis and normal maturation. No areas of osteoblastic rimming or increased cellularity were seen. The outer surface of the bone was covered on both sides by a thin layer of hypocellular fibrovascular tissue consistent with dura. These findings were consistent with a diagnosis of subdural osteoma (Fig. 4). Postoperatively, the patient did well and eventually was discharged to home with no focal neurological deficit.
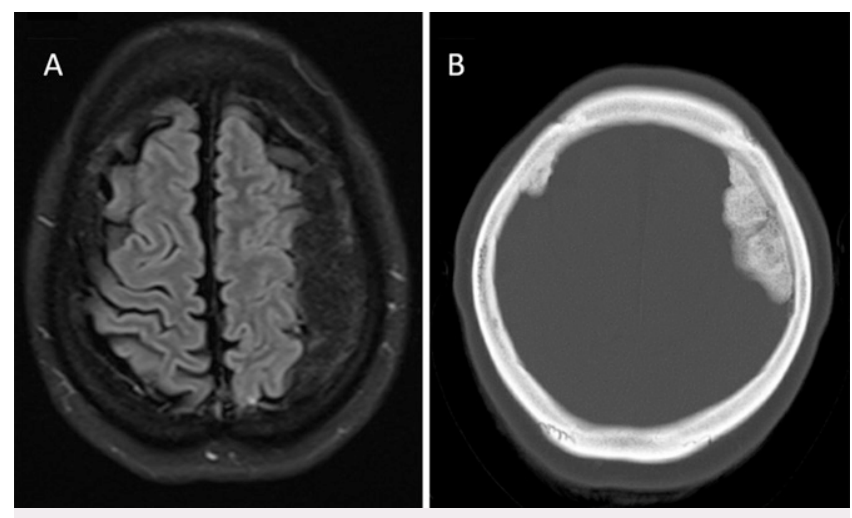

FIG. 2. Subsequent imaging revealed growth of the lesions over a 7-year period. A: Fluid-attenuated inversion recovery MRI shows increased size of the tumor, mass effect, and sulcal effacement. B: Head CT reinforces the bony character and shows growth bilaterally.

\section{Discussion}

\section{Observations}

Osteomas are benign neoplasms of bony tissue. Intracranial osteomas that are not connected to the inner table of the skull are rare. They appear bone-like on imaging, with a wide base closer to the skull protruding inward to the brain. ${ }^{2}$ Our patient had a very large, $15-\mathrm{cm}$, sheet-like intracranial subdural osteoma that was initially seen on imaging 7 years before excision, at which time it was measured on an MRI scan to be $5 \mathrm{~cm}$. The interval growth of approximately $10 \mathrm{~cm}$ over 7 years gives us an idea of the growth rate of the tumor. According to our literature review, interval growth findings, large size, and bilaterality of the tumor have not been reported before. These tumors are often hard to distinguish from other mimics on imaging, such as hyperostosis interna, extradural osteoma, or calcified or intraosseous meningioma. On a CT scan, a change in density between the tumor and the skull, as seen in Fig. 2B, may provide a hint pointing toward a submeningeal lesion, as has been described before. ${ }^{3}$ Our case also shows a similarappearing lesion on the right that was very small in size, grew a little, appeared asymptomatic at the time of diagnosis, and was not resected. This perhaps raises the possibility of a tendency of this particular patient to develop these tumors, because the significant difference in size attests to a possible different time of onset. Although our patient underwent resection of only the tumor that was asserting mass effect on the brain, we suspect that the contralateral small tumor is likely to continue to grow and require surgery in the future. A literature review revealed that all cases previously described were one-sided lesions that were overwhelmingly present in the frontal area. Some of them were connected with the dura mater, and some were intracerebral
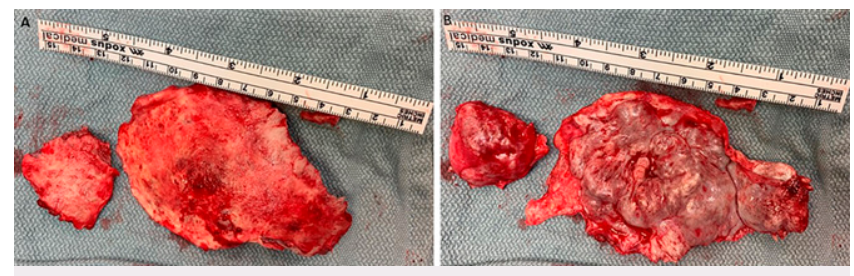

FIG. 3. Gross specimen. A: Outer surface of the large bony tumor. B: The inner surface of the tumor with imprints of gyri and sulci. 


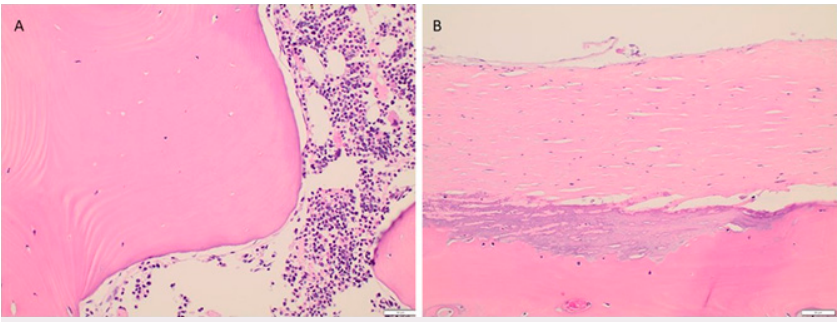

FIG. 4. Histopathology with original magnification $\times 20$. A: Mature trabecular bone with no areas of osteoblastic rimming. B: The outer surface of the lesion, covered by a thin layer of fibrovascular dura.

without connection to the dura. ${ }^{1}$ We found one report of multiple osteomas in one patient; however, they were on one side and were aggregates at one location. ${ }^{4}$ The focality of the lesion and the history of traumas in some patients have led to the hypothesis that trauma may play a role in pathogenesis. However, mostly the trigger remains unclear. The most common symptom was headache; however, some patients had vertigo, fatigue, tinnitus, and altered mental status. ${ }^{1}$ In one case described in 1983, a 16-year-old girl had presented with a seizure and was found to have an intracallosal osteoma in the frontal region protruding into the lateral ventricle. ${ }^{5}$

\section{Lessons}

Intracranial subdural osteomas are rare. Previous case reports described unilateral, mostly frontal tumors without interval imaging. We report a unique case with bilateral, radiologically similar tumors of different sizes visualized by CT and MRI 7 years apart with the large tumor being histologically confirmed to be an intracranial subdural osteoma. The imaging provides a rare glimpse of the slow growth of the tumor over the course of 7 years. Furthermore, it emphasizes that subdural osteomas may be small and inconsequential on the initial encounter, but they may grow significantly over the years and compress the subjacent brain.

\section{References}

1. Yang Y, Gu Z, Song Y. Intracranial subdural osteoma. Eur J Inflamm. Published online June 13, 2020. doi:10.1177/ 2058739220926854

2. Choudhury AR, Haleem A, Tjan GT. Solitary intradural intracranial osteoma. Br J Neurosurg. 1995;9(4):557-559.

3. Avrahami E, Even I. Osteoma of the inner table of the skull-CT diagnosis. Clin Radiol. 2000;55(6):435-438.

4. Akiyama M, Tanaka T, Hasegawa Y, et al. Multiple intracranial subarachnoid osteomas. Acta Neurochir (Wien). 2005;147(10): 1085-1089.

5. Vakaet A, De Reuck J, Thiery E, et al. Intracerebral osteoma: a clinicopathologic and neuropsychologic case study. Childs Brain. 1983;10(4):281-285.

\section{Disclosures}

The authors report no conflict of interest concerning the materials or methods used in this study or the findings specified in this paper.

\section{Author Contributions}

Conception and design: El-Zuway, Laghari. Acquisition of data: El-Zuway, Laghari. Analysis and interpretation of data: El-Zuway. Drafting the article: all authors. Critically revising the article: ElZuway, Eakin. Reviewed submitted version of manuscript: El-Zuway, Laghari. Approved the final version of the manuscript on behalf of all authors: El-Zuway. Administrative/technical/material support: ElZuway. Study supervision: El-Zuway.

\section{Correspondence}

Salem El-Zuway: University of Pittsburgh Medical Center Hamot, Erie, PA.salem_elzuway@yahoo.ca. 